\title{
Comparison of metal artifact in digital tomosynthesis and computed tomography for evaluation of phantoms
}

\author{
Tsutomu Gomi
}

School of Allied Health Sciences, Kitasato University, Sagamihara, Japan

Email: gomi@kitasato-u.ac.jp

Received 23 May 2013; revised 23 June 2013; accepted 30 June 2013

Copyright (C) 2013 Tsutomu Gomi. This is an open access article distributed under the Creative Commons Attribution License, which permits unrestricted use, distribution, and reproduction in any medium, provided the original work is properly cited.

\begin{abstract}
We compared metal artifact in $\mathrm{X}$-ray digital tomosynthesis (DT) and modern computed tomography (CT) reconstruction to improve the image quality. We compared the images of a prosthetic phantom (titanium) and a contrast-detail phantom obtained by DT using conventional filtered backprojection (FBP), metal artifact reduction (MAR) processing, and simultaneous iterative reconstruction technique (SIRT) methods and those obtained by CT using conventional FBP and adaptive statistical iterative reconstruction methods. The effectiveness of each method for enhancing the visibility of a prosthetic phantom was quantified in terms of the intensity profile and root mean square error, and the removal of ghosting artifacts was quantified in terms of the artifact spread function (ASF). In addition, low contrast resolution was evaluated in terms of the contrast-tonoise ratio. Image error was smaller in the MAR DT images in the near in-focus-plane, and the intensity profiles revealed the beam hardening effect. Streak artifacts were reduced in the SIRT DT and adaptive statistical iterative reconstruction CT images. The ASF performances of the algorithms were ranked in descending order: 1) MAR DT; 2) CT (adaptive statistical iterative reconstruction, and conventional FBP); 3) SIRT DT; and 4) conventional FBP DT. The low contrast resolution was higher in the CT images than in the DT images. In conclusion, a review of the results revealed that the metal artifact reduction was highest for tomosynthesis with MAR processing, and the low contrast resolution performance was highest for $\mathrm{CT}$.
\end{abstract}

Keywords: Tomosynthesis; Computed Tomography; Prosthesis

\section{INTRODUCTION}

Digital tomosynthesis provides the benefits of digital imaging [1-7], and the tomographic benefits of computed tomography (CT) at decreased radiation doses and cost in an approach that is easily implemented in conjunction with radiography. This technique has been investigated in angiography and in imaging of the chest, hand joints, lungs, teeth, and breasts [7,8-11]. In addition, digital tomosynthesis using the filtered back projection (FBP) algorithm has been reported to be favorably efficient [11] but its effectiveness depends considerably on the region of the image. This type of digital tomosynthesis gives results that are independent of the type of metal used in the patient [12]. In addition, flexibility in choosing digital tomosynthesis imaging parameters on the basis of the desired final images and generation of high-quality images might be beneficial. Ideally, structures in a given plane of interest should be clearly displayed in the corresponding tomosynthesis reconstruction plane, whereas structures located outside of that plane should not be visible. Practically, the limited angular range of the tomosynthesis image acquisition geometry dictates that the spatial resolution is limited in the dimension perpendicular to the detector plane; therefore, out-of-plane structures cannot be totally removed from the reconstruction plane. Out-of-plane structures are present in every reconstruction plane. At one projection angle, these ghosting features are distributed along the line formed by the Xray source and the actual feature.

Imaging by X-ray $\mathrm{CT}$ has improved over the past three decades and is now a powerful tool in medical diagnostics. It has become an essential noninvasive imaging technique since the advent of spiral CT imaging in the 1990s and has led to shorter scan times and improved three-dimensional (3D) spatial resolution. Further, CT provides high resolution in the tomographic plane but limited resolution in the axial direction. However, the quality of images generated by a CT scanner could still be compromised by the presence of metal objects in the field-of-view. The imaging of patients with metal im- 
plants such as marker pins, dental fillings, or hip prostheses, might give rise to artifacts such as bright and dark streaks, cupping, and capping. This susceptibility to artifacts could be attributed to quantum noise, scattered radiation, and beam hardening [13]. Metal artifacts influence image quality by reducing contrast and obscuring details; thus, they hinder the ability to detect structures of interest, which can possibly lead to misdiagnosis. Photon starvation could be a major cause of metal artifacts. In addition, CT values are reduced, which can lead to errors when using these data (e.g., for attenuation correction in positron emission tomography/CT imaging) [14]. The metallic components of arthroplasty devices are highcontrast objects that generate artifacts when imaged in CT scans. The presence of artifacts, along with the partial-volume effect, severely restricts the potential for objective quantification of total joint replacement by CT. Methods for reducing metal artifacts aim to improve the quality of images affected by the artifacts. Iterative [1518] and wavelet reconstruction techniques [19] as well as mathematic interpolations and combinations of filtering techniques have been studied in an attempt to further reduce metal artifacts and provide missing data obscured by them $[20,21]$.

In the presence of metallic joint prostheses or osteosynthetic materials, the metal implant itself and the interfaces between the implant, dose, and surrounding tissue should be evaluated. Important diagnostic criteria include exclusion of features or loosening and verification of sufficient coverage of the implant [22] as well as ruling out of hematoma or inflammation in the adjacent soft tissue. The CT scanning parameters that influence metal artifacts include detector element collimation, Xray beam energy (kilovolt peak), tube current (mAs), and pitch for image acquisition and the reconstruction algorithm or filter reconstruction section thickness [23]. However, because of the occurrence of metal artifacts, evaluation of these features remains challenging, with many cases rendered uninterpretable even with hard convolution kernels and widened CT density ranges [24]. In tomosynthesis, metallic artifacts occur as very low signals along the sweep direction around the edge of a highattenuation material such as a metal prosthesis or an osteosynthetic material. These artifacts are predominantly caused by reconstruction of the very low-level signals in the shadow of the highly attenuating object and, to a much lesser degree, by the limited sweep angle. Tomosynthesis has advantages over CT imaging in which either beam-hardening or metallic artifacts complicate detection of surrounding bone changes [12,25].

In this study, we compared the ability of tomosynthesis and modern CT to accurately image hip prosthesis and contrast-detail phantom, particularly with respect to metal artifact reduction performance.

\section{MATERIALS AND METHODS}

\subsection{Phantom Specifications}

For artifact evaluations, a hip prosthesis phantom (PerFix HA CMT91006; Japan Medical Materials Co., Tokyo, Japan, Figure 1) was used in a polymethyl methacrylate (PMMA) case filled with water (case $\varphi, 200 \times 300 \mathrm{~mm}$ ). The hip prosthesis phantom was positioned in the center of the case. In this study, an implant was not embedded in a bone structure within a hip prosthesis phantom because the phantom was used only to evaluate metal artifact reduction and not hip replacement loosening.

For evaluation of low contrast resolution, the contrastdetail phantom was used with PMMA slabs. Contrastdetail phantom of different diameters (artificial region, $\mathrm{CaCO}_{3}$ ) and thicknesses were arranged within the PMMA slabs (Figure 2). For X-ray imaging, we are arranged with a PMMA slabs $(200 \times 200 \mathrm{~mm})$ of $100 \mathrm{~mm}$ thickness placed on the top and bottom of the contrast-detail phantom.

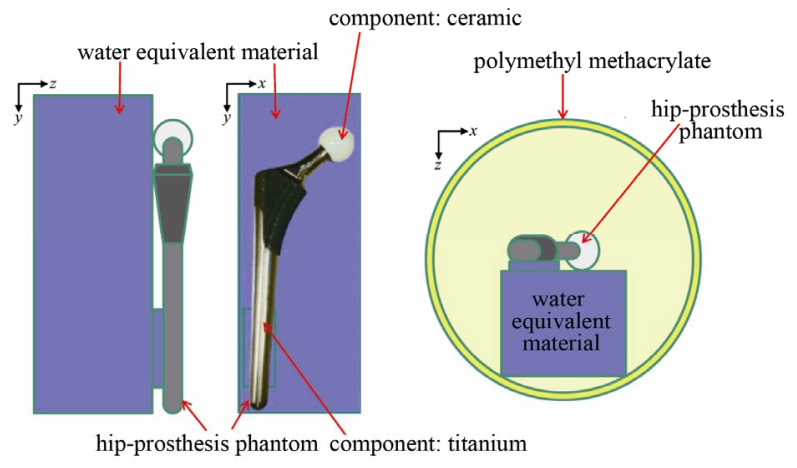

Figure 1. Illustration of the hip-prosthesis phantom used in this study.

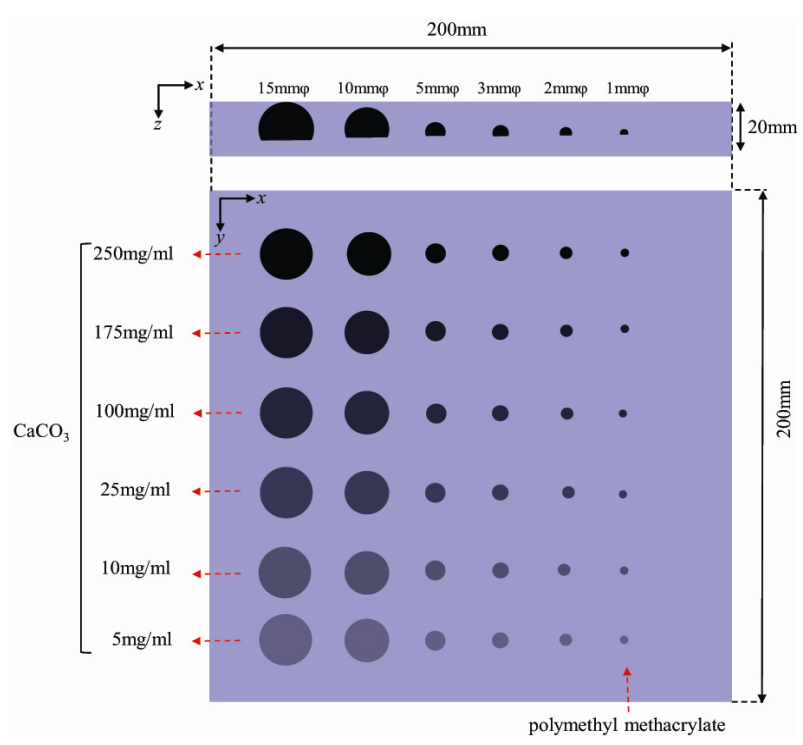

Figure 2. Illustration of the contrast-detail phantom used in this study. 
The prosthetic and contrast-detail phantoms were designed to evaluate image reconstruction quality for inplane ( $x-y$ plane) and out-plane ( $z$ axis) images.

\subsection{Tomosynthesis System}

The tomosynthesis system (Sonial Vision Safire II; Shimadzu Co., Kyoto, Japan) consisted of an X-ray tube with a $0.4-\mathrm{mm}$ focal spot and a $362.88 \times 362.88-\mathrm{mm}$ digital flat-panel detector composed of amorphous selenium. Each detector element was $150 \times 150 \mu \mathrm{m}$ in size. Tomography was performed linearly with a total acquisition time of $6.4 \mathrm{~s}$ [80 kVp, $250 \mathrm{~mA}, 20 \mathrm{~ms} / \mathrm{view}$; effective dose in accordance with the International Commission on Radiological Protection (ICRP); the anatomy used for dose calculation: X-ray pelvis, $0.69 \mathrm{mSv}$ (ICRP 103)] and an acquisition angle of $40^{\circ}$. Monte Carlo based software (PCXMC version 2.0; Radiation and Nuclear Safety Authority, Helsinki, Finland) was used to calculate the effective dose [26]. A matrix size of $1440 \times 1440$ with 12 bits per image was used to sample projection images during a single tomographic pass ( 74 projections) and was used to reconstruct tomograms of a desired height. A 4-mm slice thickness at 1-mm reconstruction intervals was used to obtain the reconstructed images $(0.272 \mathrm{~mm} /$ pixel). An antiscatter grid was used (focused type, grid ratio 12:1). The distance from the source to the isocenter was $980 \mathrm{~mm}$ and that from the isocenter to the detector was $1100 \mathrm{~mm}$ (3.0-mm aluminum equivalent filtration).

\subsection{CT System}

CT scans were performed using a multi-slice CT scanner (64-slice Discovery CT 750HD scanner; General Electric (GE) Healthcare Corp., Milwaukee, WI, USA) with settings of $120 \mathrm{kVp}, 150 \mathrm{~mA}, 0.625 \mathrm{~mm} \times 64$ collimation, "STND" reconstruction kernel, and a 1-s gantry-rotation time at a beam pitch of 0.984 [effective dose, $4.1 \mathrm{mSv}$ (ICRP 103)]. Monte Carlo based software (CT dosimetry calculator, CT-Expo version 2.1; Georg Stamm, Rosdorf, Germany) was used to calculate the effective dose [27]. The clinical task was to assess hip prostheses. A 4-mmthick slice (reformatted images) is generally used in clinical practice; therefore, in this study, reformatted reconstructed images of 4-mm-thick slices (axial images reconstructed from 1-mm-thick slices) were obtained at $1-\mathrm{mm}$ reconstruction intervals $(512 \times 512$ pixels and a 140-mm field-of-view).

\subsection{Tomosynthesis Reconstruction}

In FBP algorithms, which are widely used in tomography, many projections are acquired to reconstruct cross-sectional images. In two-dimensional (2D) tomographic imaging, projecting an object corresponds to sampling it perpendicular to the X-ray beam in the Fourier space [28]. The relationship between the radon transform and cone-beam projections has been thoroughly studied, and solutions to the cone-beam reconstruction have been provided [29]. Filtering this 2D image by multiplying its Fourier transform by a Ramp or Shepp-Logan filter kernel restores the proper impulse shape to the reconstructed image. Performing this filtering in 2D Fourier space is an acceptable method of restoring the image, but it is possible that an equivalent filtering response might be obtained much faster by filtering the one-dimensional projection data before back projecting them. The FBP algorithm generally provides highly precise 3D-reconstruction images [30]. In this study, the conventional SheppLogan filter kernel was used to reconstruct the tomosynthesis FBP images.

An iterative algorithm performs reconstruction recursively [31,32], unlike the one-step operation in backprojection and FBP algorithms. The reconstruction is accomplished by iteratively updating the unknown linear attenuation coefficients by minimizing the error between the measured and calculated projection data. The original method in this family of algebraic reconstruction techniques (ART) [33] has been determined. Because only a single projection value is used to update the linear attenuation coefficients at a time, ART has a fast convergence speed but will converge to a least squares solution, which can be very noisy for severely ill-posed inverse problems such as limited-angle tomosynthesis reconstruction. To improve the ART method, variations have been proposed regarding its implementation. Depending on the amount of projection data and the method used to update the current estimation, ART has been modified to other methods such as the simultaneous iterative reconstruction technique (SIRT) [33]. SIRT is applied iteratively so that the projections of the reconstructed volume, computed from an image formation model, resemble the experimental projections. In this study, the SIRT iterations used were 5, 10, 20, 40, 60, and 100 .

The metal artifact reduction (MAR) processing method is based on a modified Shepp-Logan reconstruction filter kernel by considering the additional weight of the direct current components (" $W$ " factor) in the frequency domain [34]. The MAR method is a filtering method that can be used in combination with the backprojection algorithm to yield sliced images with the desired properties via tomosynthesis. A modified SheppLogan reconstruction filter kernel reduces artifacts and improves the quality of images affected by metal artifacts. When MAR processing uses a large value for the $W$ factor, a near shift-and-add processing image is generated, and reduction of metal artifacts occurs. The effectiveness of the method in enhancing the visibility of a prosthetic case was quantified in terms of removal of ghosting arti- 
facts. In this study, a direct-current component was used at three levels: low $(W=0.04)$, medium $(W=0.06)$, and high $(W=0.08)$.

\subsection{CT Reconstruction}

Iterative reconstruction algorithms, with and without incorporation of a priori information, have been used to reconstruct incomplete projections [13-15,19]. Although previous results obtained by iterative reconstruction have been unsatisfactory, a recently developed iterative deblurring method has produced image reconstruction from incomplete data with few artifacts. Iterative reconstruction, which has recently become available on commercial CT scanners, enables metal artifact noise reduction without a trade-off in spatial resolution [35]. However, iterative reconstruction has unfavorable effects: its use alters the texture of the image noise, which can yield an unusually homogeneous image. This may not be immediately appealing to most radiologists, who are usually accustomed to FBP images [36]. Moreover, an excessive degree of iterative reconstruction might obscure fine and subtle details [37].

In computation with iterative reconstruction, the image has an initial set of values that are iteratively optimized according to the rules of the model. The FBP image is used for the initial condition in adaptive statistical iterative reconstruction for the following reasons: it is presumably identical to the final optimized solution (reducing the need for iterations), it is a valid indicator of the specific slice image noise, and it can be obtained rapidly. For modeling and in using iterative reconstruction, minimal convergence is achievable with adaptive statistical iterative reconstruction. However, a fully converged, $100 \%$ adaptive statistical iterative reconstruction image has a noise-free appearance with unusually homogeneous attenuation. Because some noise is inherent in CT, the use of $100 \%$ adaptive statistical iterative reconstruction might not be immediately appealing to most radiologists. However, blended images containing a linear mixture of the original FBP and this reconstruction can exhibit markedly decreased noise while retaining a more typical CT appearance. This blended image can be adjusted from $1 \%$ to $100 \%$ in adaptive statistical iterative reconstruction. We selected blending ratios of $20 \%, 40 \%$, and $60 \%$ on the basis of the results of a previous study [36], and we added an evaluation of pure iterative reconstruction (100\%). As in conventional FBP reconstruction, standard reconstruction kernels were used.

\subsection{Evaluation}

To evaluate the effects of artifacts on reconstructed image features in the in-focus-plane, we calculated an intensity profile and the root mean square error (RMSE).
To evaluate the effects of artifacts on reconstructed image features in the adjacent out-of-plane ( $z$ direction) area, we calculated an artifact spread function (ASF). The evaluations used a hip prosthesis phantom. Different reconstruction methods in the in-focus-plane were used to compare the intensity profiles. Wu et al. have proposed an ASF metric to quantify the artifacts observed in planes outside the focus image plane [38]. These artifacts are generated from real features located in the focus image plane and resemble real features. The capability of tomosynthesis in differentiating features that are superimposed along the $z$ direction can be reflected from the ASF measurement. The artifacts exhibited in the image plane are defined by ASF as:

$$
A S F=\frac{N_{\text {artifact }}(z)-N_{B G}(z)}{N_{\text {artifact }}\left(z_{0}\right)-N_{B G}\left(z_{0}\right)}
$$

where $z_{0}$ and $z$ are the locations of the real features in the in-focus and out-of-planes, respectively. $N_{\text {artifact }}\left(z_{0}\right)$ and $N_{B G}\left(z_{0}\right)$ are the region of interest (ROI) for average pixel intensities of the feature and the image background in the in-focus-plane, respectively. $N_{\text {artifact }}(z)$ and $N_{B G}(z)$ are the ROI for average pixel intensities of the artifact and image background in the out-of-plane, respectively. The ROI size for evaluation was chosen as a $20 \times 20$-pixel region for all features. Areas of measurement for the ASF metric are displayed in Figure 3. Another important metric to be considered is RMSE, which can be computed by obtaining the root of the summation of the square of the standard deviation and the square of the bias. The reference image is a straight projected radiographic image. Different reconstruction methods in the in-focus-plane (an image that is the same as the intensity profile evaluation image) were used to compare RMSEs. For CT (mid-plane reformatted image) comparison with the projected radiographic image, we coordinated the magnification (scaling processing) of the projected radiographic image. The errors in the image plane are defined in terms of RMSE as follows:

$$
\mathrm{RMSE}=\sqrt{\sum_{i=1}^{n}\left(X-x_{i}\right)^{2} / n}
$$

Where $X$ is the observed image, $x_{i}$ is the reference image, and $n$ is the number of compounds in the analyzed set.

To quantitatively evaluate the reconstructed image quality (low contrast resolution), we calculated the image contrast derived from the contrast-to-noise ratio (CNR) of selected features to determine the low contrast detectability (region: $\varphi, 15 \mathrm{~mm} ; 100 \mathrm{mg} / \mathrm{ml} \mathrm{CaCo}_{3}$ ) at its infocus-plane for detection of detail bony changes. The evaluation used a contrast-detail phantom. CNR was defined as follows: 

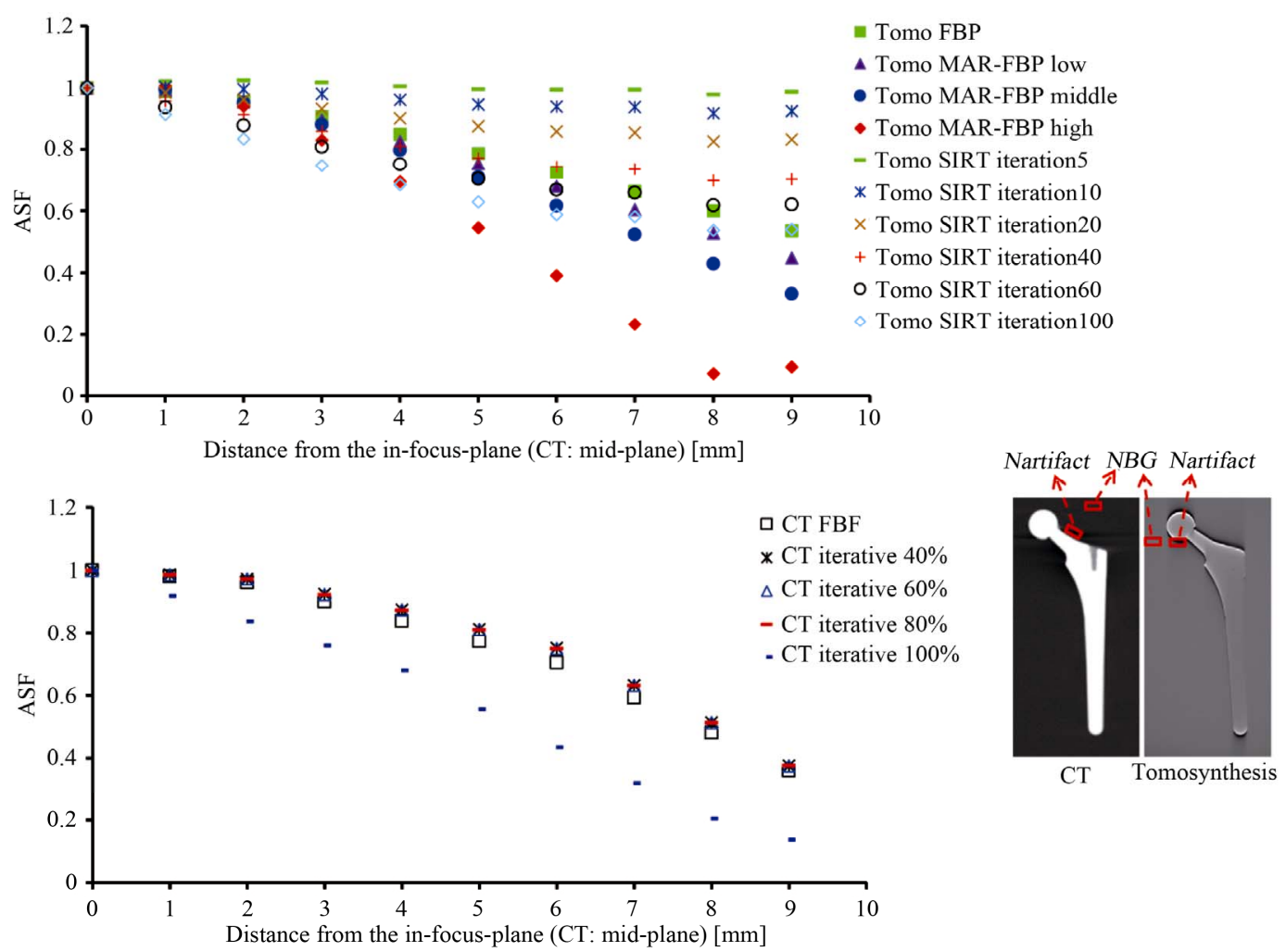

Figure 3. Comparison between artifact-spread function (ASF) versus distance from the in-focus-plane for tomosynthesis and CT. Slices with positive distance are above the feature prosthesis. The ASF were obtained by averaging three repeated measurements. The prosthetic was in the field of view, $9 \mathrm{~mm}$ from in-focus-plane.

$$
C N R=\frac{N_{1}-N_{0}}{\sigma_{0}}
$$

where $N_{1}$ is the mean pixel value in the object, $N_{0}$ is the mean pixel value in the background area, and $\sigma_{0}$ is the standard deviation of the pixel values in the background. The parameter $\sigma_{0}$ not only includes photon statistics and electronic noise in the results but also structural noise that can obscure the object. Areas of measurement for the CNR metric are presented in Figure 4.

For tomosynthesis acquisition, the phantom was arranged in parallel to the $x-y$ detector plane. For CT acquisition, the phantom was arranged perpendicular to the $x-y$ detector plane; coronal reformatted images were used as evaluation images.

\section{RESULTS}

The reconstruction images of the prosthetic phantom obtained using the conventional FBP, MAR processing, SIRT, and adaptive statistical iterative methods are presented in Figure 5. With regard to the tomosynthesis images, the results revealed that the MAR processing and SIRT methods produced reconstructed images of features that did not contain metal artifacts in the vertical direction (X-ray sweep direction). In particular, reduction of artifacts was evident in the caput femoris and peripheral

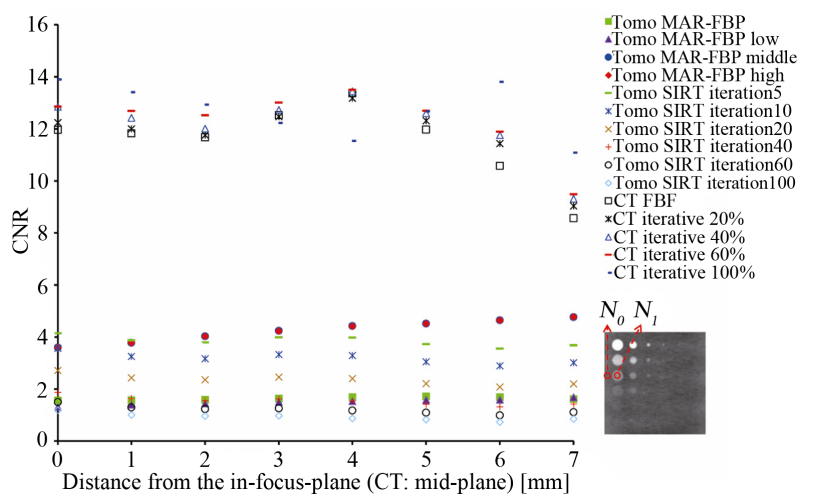

Figure 4. Comparison between contrast-to-noise ratio (CNR) versus distance from the in-focus-plane for tomosynthesis and CT. Slices with positive distance are above the feature signal. The CNR were obtained by averaging three repeated measurements. The contrast-detail phantom was in the field of view, $7 \mathrm{~mm}$ from in-focus-plane.

regions of the prosthetic phantom. Furthermore, with regard to the CT images, the results could not confirm that conventional FBP and adaptive statistical iterative reconstruction methods could produce reconstructed images of features that were free of metal artifacts in the horizontal direction (X-ray sweep direction).

The intensity profile of the femoral neck for the conventional FBP, MAR processing, SIRT, and adaptive statis- 

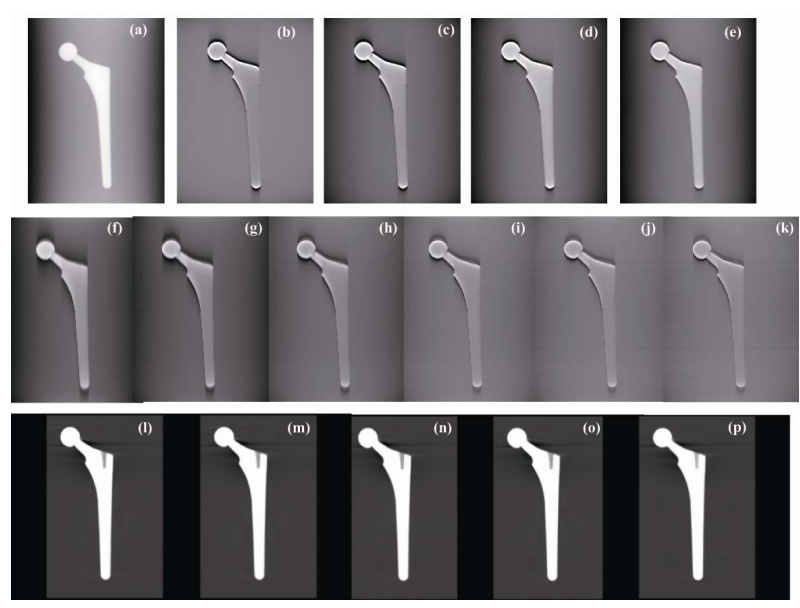

Figure 5. Comparison between tomosynthesis, CT images and those obtained from the imaging algorithms of metal-artifactreduction (MAR) processing, conventional filtered back projection (FBP), simultaneous iterative reconstruction technique (SIRT), and adaptive statistical iterative reconstruction in the in-focus-plane. (a) projection; (b) FBP; (c) MAR low; (d) MAR middle; (e) MAR high; (f) SIRT iteration 5; (g) SIRT iteration 10; (h) SIRT iteration 20; (i) SIRT iteration 40; (j) SIRT iteration 60; (k) SIRT iteration 100; (l) CT FBP; (m) CT iterative $20 \%$; (n) CT iterative $40 \%$; (o) CT iterative 60\%; (p) CT iterative $100 \%$. The FBP, MAR, SIRT tomosynthesis images for the corresponding prosthesis phantom are displayed with the same window width and window level, whereas the CT image of the same prosthesis phantom is displayed with narrower window width and different window level to archive visually comparable contrast and background gray level. The $\mathrm{X}$-ray source moved in the vertical direction relative to the image shown.

tical iterative reconstruction methods have been presented in Figure 6. We set up the comparison to evaluate the regions of the femoral neck in which artifacts were observed to occur in both techniques. Tomosynthesis and $\mathrm{CT}$ profiles were set at 30 pixels (profile length, $25 \mathrm{~mm}$ ). In the tomosynthesis images, MAR processing and SIRT methods reduced metal artifacts. Improvement of image quality by reduction of artifacts (related to signal undershoot) by MAR processing and SIRT methods has been accepted. In the CT images, the conventional FBP and adaptive statistical iterative reconstruction methods produced very similar results for the selected features; the reason could be that the adaptive statistical iterative reconstruction method was developed for noise reduction and not metal artificial image reduction in high-attenuation objects. Taken together, these results indicated that MAR processing, SIRT, conventional FBP (CT), and adaptive statistical iterative reconstruction methods were effective in reducing the portion of metal artifacts resulting due to signal undershoot.

The reference (projected radiographic) reconstruction error images of the prosthetic phantom obtained using the conventional FBP (tomosynthesis), MAR processing,
SIRT, conventional FBP (CT), and adaptive statistical iterative reconstruction methods are presented in Figure 7. The tomosynthesis error images and RMSE values (Table 1) revealed that the MAR processing method reduced metal artifacts in the vertical direction (X-ray sweep direction). Reduction of artifacts was particularly apparent in the caput femoris and peripheral regions of the prosthetic phantom. The tomosynthesis error images and RMSE values revealed that the SIRT method did not reduce the incidence of metal artifacts in the vertical direction (X-ray sweep direction). In addition, these results indicate that the improvement in reduction of artifacts was not related to increase in the number of iterations in the SIRT method. The CT error images and RMSE values indicated that the conventional FBP and adaptive statistical iterative reconstruction methods produced very similar results for the selected features. The RMSE values were larger for the $\mathrm{CT}$ images than for the tomosynthesis images, which might have been because of the large influence of the femoral neck on the generated metal artifacts (X-ray sweep direction). In addition, we believe that the DT RMSE results were lower because of apparent freedom from metal artifacts because of inherent blur in the tomosynthesis reconstruction from few projections.

The chart in Figure 3 presents the ROI position and ASF results for the prosthetic phantom. The chart demonstrates that tomosynthesis with MAR processing ( $W=$ high) removed the highest number of metal artifacts. In tomosynthesis imaging, the artifact reduction images demonstrated better image quality for MAR processing than for conventional FBP using the iterative algorithm. On the other hand, in CT imaging, the artifact reduction images had better image quality for the pure iterative reconstruction algorithm (100\% adaptive statistical iterative reconstruction) than for conventional FBP using $20 \%, 40 \%$, and $60 \%$ adaptive statistical iterative reconstruction.

A comparison of the metal artifact evaluations on the basis of the image quality, intensity profiles, RMSEs, and ASFs of the tomosynthesis reconstructed images with MAR processing and the iterative algorithm and the CT reconstructed images with $100 \%$ adaptive statistical iterative reconstruction revealed decreased metal and beamhardening artifacts. Evaluation of the metal artifact evaluations through the entire thickness of the specimen revealed that the metal artifact reduction performance of the algorithm could be ranked in the descending order: 1) tomosynthesis (MAR processing, $W=$ high); 2) CT (100\% adaptive statistical iterative reconstruction); 3 ) CT (20\%, $40 \%$, and $60 \%$ adaptive statistical iterative reconstruction and conventional FBP); 4) tomosynthesis (iterative algorithm and 100 iterations); and 5) tomosynthesis (conventional FBP). 

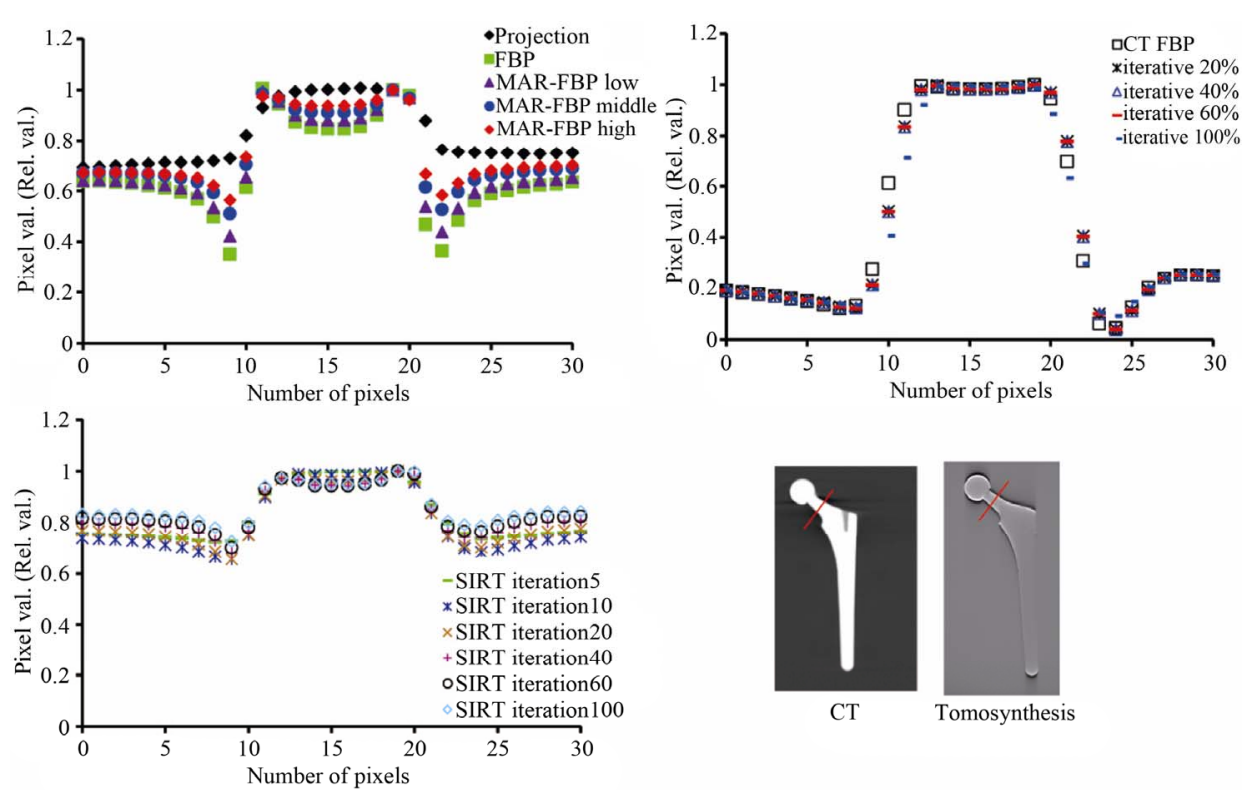

Figure 6. Comparison between intensity profiles using tomosynthesis and CT in the in-focusplane. The pixel value was normalized by removing the mean of each intensity profile. The intensity profile were obtained by averaging three repeated measurements.

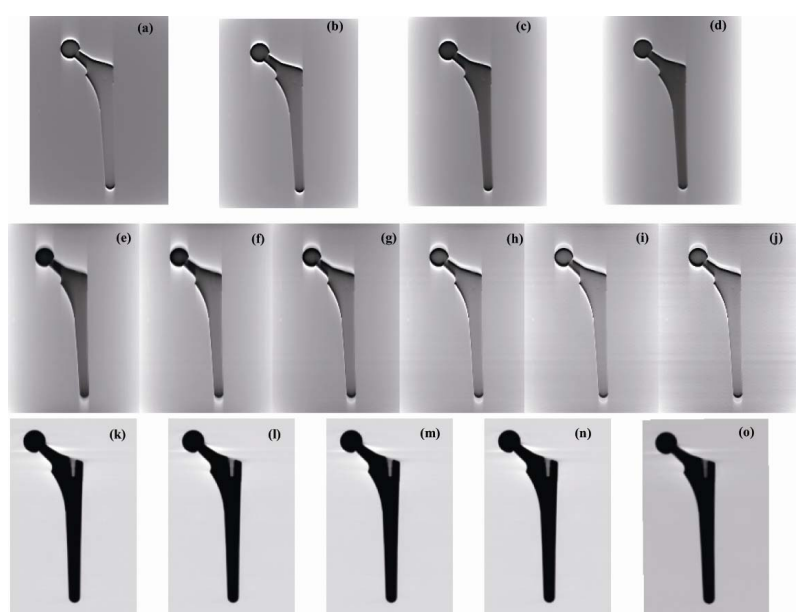

Figure 7. Comparison between tomosynthesis, CT error images and the root-mean-square-error (RMSE) of the images obtained from the imaging algorithms of MAR processing ( $W$ = low, medium, and high), conventional FBP, SIRT, and adaptive statistical iteration reconstruction in the in-focus-plane. (a) projection-FBP; (b) projection-MAR low; (c) projectionMAR middle; (d) projection-MAR high; (e) projectionSIRT iteration 5; (f) projection-SIRT iteration 10; (g) projection-SIRT iteration 20; (h) projection-SIRT iteration 40; (i) projection-SIRT iteration 60; (j) projection-SIRT iteration 100; (k) projection-CT FBP; (l) projection-CT iterative $20 \%$; (m) projection- $\mathrm{CT}$ iterative $40 \%$; (n) projection- $\mathrm{CT}$ iterative $60 \%$; (o) projection-CT iterative $100 \%$. The FBP, MAR, SIRT tomosynthesis error images for the corresponding prosthesis phantom are displayed with the same window width and window level, whereas the CT error image of the same prosthesis phantom is displayed with narrower window width and different window level to archive visually comparable contrast and background gray level. The X-ray source moved in the vertical direction relative to the image shown.
Table 1. Comparison of the root mean square errors (RMSEs) for tomosynthesis and CT. RMSEs were calculated from the in-focus-plane tomosynthesis and CT images. (DT: digital tomosynthesis).

\begin{tabular}{cc}
\hline Analysis object & RMSE \\
\hline projection-DT FBP & 0.1803 \\
projection-DT MAR low & 0.1434 \\
projection-DT MAR middle & 0.1097 \\
projection-DT MAR high & 0.0756 \\
projection-DT SIRT iteration 5 & 0.1830 \\
projection-DT SIRT iteration 10 & 0.1631 \\
projection-DT SIRT iteration 20 & 0.1834 \\
projection-DT SIRT iteration 40 & 0.2091 \\
projection-DT SIRT iteration 60 & 0.2170 \\
projection-DT SIRT iteration 100 & 0.2268 \\
projection-CT FBP & 0.3515 \\
projection-CT iterative 20\% & 0.3515 \\
projection-CT iterative 40\% & 0.3515 \\
projection-CT iterative 60\% & 0.3516 \\
projection-CT iterative $100 \%$ & 0.0543 \\
\hline
\end{tabular}

CNRs for tomosynthesis and CT were investigated, as displayed in Figure 4. Low contrast resolution was higher in the CT images than in the DT images. Low contrast characteristics of the $\mathrm{CT}$ produced an increase in the in-focus-plane CNR values but a reduction in the outof-plane CNR values. The partial volume effect in the out-of-plane images attenuated the signal components in the $z$ direction of the object. Furthermore, in the tomo- 
synthesis images, the low contrast resolution was high with MAR processing ( $W$-value set to middle and high) relative to conventional FBP and SIRT methods. Tomosynthesis images of the contrast-detail phantom produced an increase in the out-of-plane CNR values but a reduction in the in-focus-plane CNR values. In this case, we speculate that the out-of-plane images were smoothed by the discrete blur structure shadows. However, MAR processing tomosynthesis demonstrated its greatest advantages during the tomosynthesis reconstruction methods.

A review of the results revealed that metal artifact reduction was highest for tomosynthesis with MAR processing, and low contrast resolution performance was highest for CT.

\section{DISCUSSION}

Digital tomosynthesis with MAR processing was effective for reducing metal artifacts from metal objects, particularly for artifacts at 9-mm distances, but the effectiveness strongly depended on the image region. 3D visualization of the prosthetic phantom on images with MAR processing was superior to images processed by conventional FBP, iterative algorithms, and adaptive statistical iterative reconstruction.

In general, the observed artifacts are caused by the loss of the largest normal contribution from artifact-free voxels in the image. Such a voxel originally has normal contributions, and its value is slightly reduced after the largest normal contribution is omitted. A voxel with one abnormal contribution rejects this contribution while using all the others, including the largest normal contribution. Therefore, these voxels tend to have higher values than their neighboring artifact-free voxels such that artifactual needles appear when the voxels are more noticeable in the background formed from the artifact-free voxels. This is a drawback of the FBP method, but artifacts because of this effect are very evident compared with those in images without artifact reduction.

In comparison digital tomosynthesis and CT reported previously, digital tomosynthesis gives good results independent of the type of the metal present in the patient and effectively removes noise artifacts, especially at greater distances from the metal objects [12]. In this study, the results revealed that metal artifact reduction was highest for tomosynthesis with MAR processing, low contrast resolution performance was highest for CT. Flexibility in selecting the imaging parameters in digital tomosynthesis with MAR processing on the basis of the desired final images and realistic imaging conditions may be beneficial.

CT using in-focus-plane images is best for CNR optimization, whereas CT using out-of-plane images is best for CNR optimization. Furthermore, with CT, details inside the hip prosthesis phantom are clearly visible, but they do not appear in the tomosynthesis images. It appears that the number of projections, effective dose, and pixel size of the detector provide much better results for CT than for tomosynthesis. The information contained in a tomosynthesis voxel can be obtained from 74 projections. Ideally, the number of X-ray quanta for reconstruction of this voxel equals that from a single projection acquired with the same amount of total exposure. Considering the detector noise from the acquisition of each projection, CNR is reduced in tomosynthesis imaging, which distributes the effective dose over 74 projections. The effects of projection angle, which decreases the photon transmission and reduces the effective detector pixel size, further reduce CNR as well.

At a given projection angle, a high-attenuation feature can create artifacts in any voxel along the lines between the X-ray source and feature pixels in the projection. Artifact reduction becomes more difficult as the size of the high-attenuation feature increases or the distance between the voxel and high-attenuation feature decreases. The adaptive statistical iterative reconstruction technique does not assume that noise is evenly distributed across the entire image. Instead, matrix algebra is used to selectively identify and then subtract noise from the image using a mathematical model [36]. The result is a less noisy image, which is an unexpected effect for artificial image reduction.

Periprosthetic bone is clearly depicted with modern CT images that have been optimized to reduce metal artifacts [39]. The isotropic datasets of multislice CT scanners allow the reformatting of high-resolution images in any desired plane so that $3 \mathrm{D}$ anatomical relationships can be clearly depicted. However, CT images depend on numerous factors such as the size, shape, density, atomic number, and position of the metal objects along with the size and shape of the object's cross section. Particularly for small implants manufactured from relatively light metals (e.g., titanium), the impact of beam hardening and scatter effects is low; hence, distorted CT values can be neglected, and noise-induced streaking artifacts primarily affect the image quality. In these cases, the pure iterative reconstruction algorithm appears to be a promising approach to reduce the artifacts stemming from metals with relatively high atomic numbers. The metal from implant hardware produces streaks and other artifacts on CT scans, thereby adversely affecting image quality. These artifacts are affected by factors related to both hardware and scanning parameters [detector element collimation, X-ray beam energy (kilovolt peak), tube current (mAs), and pitch for image acquisition as well as to the reconstruction algorithm or filter reconstruction section thickness $[23,39]$.

In conclusion, the application of digital tomosynthesis 
with MAR processing method could considerably improve the quality of images distorted by metal artifacts. Digital tomosynthesis with MAR processing provides higher quality (reducing of beam hardening effect, and streak artifacts) of metal artifact reduction images compared with those obtained by CT (only the adaptive statistical iterative and conventional FBP reconstruction technique for modern GE scanners). On the other hand, low contrast resolution was higher in CT images than in tomosynthesis images.

\section{REFERENCES}

[1] Ziedses des Plante, B.G. (1932) Eine neue methode zur differenzierung in der roentgenographie (planigraphie). Acta Radiologica, 13, 182-192. doi:10.3109/00016923209135135

[2] Miller, E.R., McCurry, E.M. and Hruska, B. (1971) An infinite number of laminagrams from a finite number of radiographs. Radiology, 98, 249-255.

[3] Grant, D.G. (1972) Tomosynthesis. A three-dimensional radiographic imaging technique. IEEE Transaction on Biomedical Engineering, 19, 20-28. doi:10.1109/TBME.1972.324154

[4] Baily, N.A., Lasser, E.C. and Crepeau, R.L. (1973) Electrofluoro-plangigraphy. Radiology, 107, 669-671.

[5] Kruger, R.A., Nelson, J.A., Ghosh-Roy, D., Miller, F.J., Anderson, R.E. and Liu, P.Y. (1983) Dynamic tomographic digital subtraction angiography using temporal filteration. Radiology, 147, 863-867.

[6] Sone, S., Kasuga, T., Sakai, F., Aoki, J., Izuno, I. and Tanizaki, Y. (1991) Development of a high-resolution digital tomosynthesis system and its clinical application. $R a-$ diographics, 11, 807-822.

[7] Sone, S., Kasuga, T., Sakai, F., Kawai, T., Oguchi, K. and Hirano, H. (1995) Image processing in the digital tomosynthesis for pulmonary imaging. European Radiol$o g y$, 5, 96-101. doi:10.1007/BF00178089

[8] Stiel, G., Stiel, L.G., Klotz, E. and Nienaber, C.A. (1993) Digital flashing tomosynthesis: A promising technique for angiographic screening. IEEE Transaction on Medical Imaging, 12, 314-321. doi:10.1109/42.232261

[9] Duryea, J., Dobbins, J.T. and Lynch, J.A. (2003) Digital tomosynthesis of hand joints for arthritis assessment. $\mathrm{Me}$ dical Physics, 30, 325-333. doi:10.1118/1.1543573

[10] Niklason, L.T., Christian, B.T., Niklason, L.E., Kopans, D.B., Castleberry, D.E. and Opsahl-Ong, B.H. (1997) Digital tomosynthesis in breast imaging. Radiology, 205, 399-406.

[11] Dobbins III, J.T. and Godfrey, D.J. (2003) Digital x-ray tomosynthesis: Current state of the art and clinical potential. Physics in Medicine and Biology, 48, R65-R106. doi:10.1088/0031-9155/48/19/R01

[12] Gomi, T. and Hirano, H. (2008) Clinical potential of digital linear tomosynthesis imaging of total joint arthroplasty. Journal of Digital Imaging, 21, 312-322. doi:10.1007/s10278-007-9040-9
[13] Joemai, R.M.S., Bruin, P.W., Veldkamp, W.J.H. and Geleijns, J. (2012) Metal artifact reduction for CT: Development, implementation, and clinical comparison of a generic and a scanner-specific technique. Medical Physics, 39, 1125-1132. doi:10.1118/1.3679863

[14] Hsieh, J. (1995) Computed tomography technology and applications; image artifacts causes and correction. In: Goldman, L.W. and Fowlkes, J.B., Eds., Medical CT and Ultrasound, Advanced Medical Publishing, Madison, 487518.

[15] Wang, G., Snyder, D.L., O’Sullivan, J.A. and Vannier, M.W. (1996) Iterative debluring for metal artifacts reduction. IEEE Transaction on Medical Imaging, 15, 657-664. doi:10.1109/42.538943

[16] Wang, G., Vannier, M.W. and Cheng, P.C. (1999) Iterative X-ray cone-beam tomography for metal artifacts reduction and local region reconstruction. Microscopy and Microanalysis, 5, 58-65. doi:10.1017/S1431927699000057

[17] Wang, G., Frei, T. and Vannier, M.W. (2000) A fast iterative algorithm for metal artifact reduction in $\mathrm{x}$-ray $\mathrm{CT}$. Academic Radiology, 7, 607-614. doi:10.1016/S1076-6332(00)80576-0

[18] De Man, B., Nuyts, J., Dupont, P. and Suetens, P. (2000) Reduction of metal streak artifacts in X-ray computed tomography using a transmission maximum a posteriori algorithm. IEEE Transaction on Nuclear Sciences, 47, 977-981. doi:10.1109/23.856534

[19] Zhao, S., Robertson, D.D., Wang, G., Whiting, B. and Bae, K.T. (2000) X-ray CT metal artifact reduction using wavelets: An application for imaging total hip prostheses. IEEE Transaction on Medical Imaging, 19, 1238-1247. doi:10.1109/42.897816

[20] Watzke, O. and Kalender, W.A. (2004) A pragmatic approach to metal artifact reduction in CT: Merging of metal artifact reduced images. European Radiology, 14, 849-856. doi:10.1007/s00330-004-2263-y

[21] Kamel, E.M., Burger, C., Buck, A., von Schulthess, G.K. and Goerres, G.W. (2003) Impact of metallic dental implants on CT-based attenuation correction in a combined PET/CT scanner. European Radiology, 13, 724-728.

[22] White, L.M. and Buckwalder, K.A. (2002) Technical considerations: CT and MR imaging in the postoperative orthopaedic patient. Seminars in Musculoskeletal Radiology, 6, 5-17. doi: $10.1055 / \mathrm{s}-2002-23160$

[23] Buckwalter, K.A., Parr, J.A., Choplin, R.H. and Capello, W.N. (2006) Multichannel CT imaging of orthopaedic hardware and implants. Seminars in Musculoskeletal Radiology, 10, 86-97. doi:10.1055/s-2006-934219

[24] Lee, M.J., Kim, S., Lee, S.A., Song, H.T., Huh, Y.M. and Kim, D.H. (2007) Overcoming artifacts from metallic orthopaedic implants at high-field-strength MR imaging and multi-detector CT. Radiographics, 27, 791-803. doi:10.1148/rg.273065087

[25] Machida, H., Yuhara, T., Mori, T., Ueno, E., Moribe, Y. and Sabol, J.M. (2010) Optimizing parameters for flatpanel detector digital tomosynthesis. Radiographics, 30, 546-562. doi:10.1148/rg.302095097 
[26] Tapiovaara, M. and Siiskonen, T. (2008) A Monte Carlo program for calculating patient doses in medical $\mathrm{x}$-ray examinations. 2nd Edition, STUK-A231, Helsinki.

[27] Stamm, G. (2012) CT-expo. http://www.sascrad.com/page10.php

[28] Kak, A. and Slaney, M. (1988) Principles of computerized tomographic imaging. IEEE, New York.

[29] Smith, D.B. (1985) Image reconstruction from cone-beam projections: Necessary and sufficient conditions and reconstruction methods. IEEE Transaction on Medical Imaging, M1-4, 14-25. doi:10.1109/TMI.1985.4307689

[30] Feldkamp, L.A., Davis, L.C. and Kress, J.W. (1984) Practical cone-beam algorithm. Journal of the Optical Society of America, A1, 612-619. doi:10.1364/JOSAA.1.000612

[31] Ruttimann, U., Groenhuis, R. and Webber, R. (1984) Restoration of digital multilane tomosynthesis by a constrained iteration method. IEEE Transaction on Medical Imaging, MI-3, 141-148. doi:10.1109/TMI.1984.4307670

[32] Bleuet, P., Guillemaud, R., Magin, I., Magnin, I. and Desbat, L. (2001) An adapted fan volume sampling scheme for $3 \mathrm{D}$ algebraic reconstruction in linear tomosynthesis. IEEE Transaction on Nuclear Sciences, 3, 1720-1724. doi:10.1109/NSSMIC.2001.1008674

[33] Gordon, R., Bender, R. and Hermen, G.T. (1970) Algebraic reconstruction techniques (ART) for three-dimensional electron microscopy and X-ray photography. Journal of theoretical biology, 29, 471-481. doi:10.1016/S0022-5193(70)80010-8

[34] Gomi, T., Hirano, H. and Umeda, T. (2009) Evaluation of the X-ray digital linear tomosynthesis reconstruction pro- cessing method for metal artifact reduction. Computerized Medical Imaging and Graphics, 33, 257-274.

doi:10.1016/j.compmedimag.2009.01.004

[35] Marin, D., Nelson, R.C., Schindera, S.T., Richard, S., Youngblood, R.S. and Yoshizumi, T.T. (2010) Low-tubevoltage, high-tube-current multidetector abdominal CT: Improved image quality and decreased radiation dose with adaptive statistical iterative reconstruction algorithm-initial clinical experience. Radiology, 254, 145-153. doi:10.1148/radiol.09090094

[36] Hara, A.K., Paden, R.G., Silva, A.C., Kujak, J.L., Lawder, H.J. and Pavlicek, W. (2009) Iterative reconstruction technique for reducing body radiation dose at CT. American Journal of Roentgenology, 193, 764-771. doi:10.2214/AJR.09.2397

[37] Yanagawa, M., Honda, O., Yoshida, S., Kikuyama, A., Inoue, A. and Sumikawa, H. (2010) Adaptive statistical iterative reconstruction technique for pulmonary $\mathrm{CT}$ : Image quality of the cadaveric lung on standard and reduced-dose CT. Academic Radiology, 17, 1259-1266. doi:10.1016/j.acra.2010.05.014

[38] Wu, T., Stewart, A., Stanton, M., McCauley, T., Phillips, W. and Kopans, D.B. (2003) Tomographic mammogramphy using a limited number of low-dose cone-beam projection images. Medical Physics, 30, 365-380. doi:10.1118/1.1543934

[39] Roth, T.D., Maertz, N.A., Parr, J.A., Buckwalter, K.A. and Choplin, R.H. (2012) CT of the hip prosthesis: Appearance of components, fixation, and complications. Radiographics, 32, 1089-1107. doi:10.1148/rg.324115183 